TAIWANESE JOURNAL OF MATHEMATICS

Vol. 9, No. 3, pp. 477-488, September 2005

This paper is available online at http://www.math.nthu.edu.tw/tjm/

\title{
BRAIDED CROSSED MODULES AND REDUCED SIMPLICIAL GROUPS
}

\author{
Z. Arvasi, M. Koçak and E. Ulualan
}

\begin{abstract}
In this paper, we established an equivalence between the category of Braided Crossed Modules of Groups and the category of Simplicial Groups with Moore Complex of length 2.
\end{abstract}

\section{INTRODUCTION}

R. Brown and N.D. Gilbert introduced in [2] the notion of braided, regular crossed modules for an algebraic models of 3-types. They then showed that this structure is closely related to simplicial groups; they proved that the category of braided, regular crossed modules is equivalent to that of simplicial groups with Moore complex of length 2.

D. Counduche has shown in [6] that the category of simplicial groups with Moore complex of lenght 2 is also equivalent to that of 2 -crossed modules. This gives a composite equivalence between the category of 2-crossed module and that of braided regular crossed modules. The related ideas of Counduche (cf.[6]) have been used by Carrasco and Cegarra [5], to study braided categorical groups. The categorical braiding is a commutator degenerate elements and the satisfaction of axioms for a braiding corresponding to the vanishing of certain commutators of intersections of face-map kernels.

In this paper we will concentrate on the reduced case: As crossed modules of groups is regular, we proved that the category of braided crossed modules of groups is equivalent to that of reduced simplicial groups with Moore complex of lenght 2 in terms of hypercrossed complex pairings $F_{\alpha, \beta}$ defined in [10].

Thus the braiding group can be given by using hypercrossed complex pairings $F_{\alpha, \beta}$ which gives products of commutators. This is reformulation of a result of Brown and Gilbert (cf. [2]). Our aim is to show the role of the $F_{\alpha, \beta}$ in the structure.

Received August 6, 2002; Accepted January 14, 2004.

Communicated by Pjek-Hwee Lee.

2000 Mathematics Subject Classification: 18D35, 18G30, 18G50, 18 G55.

Key words and phrases: Crossed modules, Simplicial groups, Moore Complex. 


\section{PRELIMINARIES}

\subsection{Truncated Simplicial Groups}

Denoting the usual category of finite ordinals by $\Delta$, we obtain for each $k \geq 0$, a subcategory $\Delta_{\leq k}$ determined by the objects [j] of $\Delta$ with $j \leq k$. A simplicial group is a functor from the opposite category $\Delta^{o p}$ to the category of groups Grp. A reduced simplicial group is a simplicial group which last component is trivial. A $k$-truncated simplicial group is a functor from $\Delta_{\leq k}^{o p}$ to Grp. We denote the category of simplicial groups by SimpGrp and the category of $k$-truncated simplicial groups by $\operatorname{Tr}_{k} \operatorname{SimpGrp~.~By~a~} k$-truncation of a simplicial group, we mean a $k$-truncated simplicial group $\operatorname{tr}_{k} \mathbf{G}$ obtained by forgetting dimensions of order $>$ in a simplicial group G. This gives a truncation functor $\operatorname{tr}_{k}: \operatorname{SimpGrp} \rightarrow \operatorname{Tr}_{k} \operatorname{SimpGrp}$ which admits a right adjoint $\operatorname{cosk}_{k}: \operatorname{Tr}_{k} \operatorname{SimpGrp} \rightarrow$ SimpGrp called the $k$-coskeleton functor, and a left adjoint $\mathbf{s k}_{k}: \operatorname{Tr}_{k} \operatorname{SimpGrp} \rightarrow$ SimpGrp , called the $k$-skeleton functor. For explicit constructions of these see [7].

Recall that given a simplicial group $\mathbf{G}$, the Moore complex $(\mathbf{N G}, \partial)$ of $\mathbf{G}$ is the normal chain complex defined by

$$
(\mathbf{N G})_{n}=\bigcap_{i=0}^{n-1} \operatorname{ker} d_{i}^{n}
$$

with $\partial_{n}: N G_{n} \rightarrow N G_{n-1}$ induced from $d_{n}^{n}$ by restriction. There is an alternative form of Moore complex given by the convention of taking

$$
\bigcap_{i=1}^{n} \operatorname{ker} d_{i}^{n}
$$

and using $d_{0}$ instead of $d_{n}$ as the boundary. They lead to equivalent theories.

The $\mathrm{n}^{\text {th }}$ homotopy group $\pi_{n}(\mathbf{G})$ of $\mathbf{G}$ is the $\mathrm{n}^{\text {th }}$ homology of the Moore complex of $\mathbf{G}$, i.e.

$$
\begin{aligned}
\pi_{n}(\mathbf{G}) & \cong H_{n}(\mathbf{N G}, \partial) \\
& =\bigcap_{i=0}^{n} \operatorname{ker} d_{i}^{n} / d_{n+1}^{n+1}\left(\bigcap_{i=0}^{n} \operatorname{ker} d_{i}^{n+1}\right)
\end{aligned}
$$

We say that the Moore complex NG of a simplicial group is of length $k$ if $N G_{n}=1$ for all $n \geq k+1$, so that a Moore complex of length $k$ is also of length $l$ for $l \geq k$.

Corollary 2.1. Let $\boldsymbol{G}^{\prime}$ be $(n-1)$-truncated simplicial group. Then there is a simplicial group $\boldsymbol{G}$ with $\operatorname{tr}_{k} \boldsymbol{G} \simeq \boldsymbol{G}^{\prime}$ if and only if $\boldsymbol{G}^{\prime}$ satisfies the following property: 
For all nonempty sets of indices $(I \neq J), I, J \subset[n-1]$ with $I \cup J=[n-1]$,

$$
\left[\bigcap_{i \in I} \operatorname{kerd}_{i}, \bigcap_{j \in J} \operatorname{kerd}_{j}\right]=1
$$

\subsection{Peiffer Pairings Generate}

In the following we will define a normal subgroup $N_{n}$ of $G_{n}$. First of all we adapt ideas from Carrasco $[3,4]$ to get the construction of a useful family of natural pairings. We define a set $P(n)$ consisting of pairs of elements $(\alpha, \beta)$ from $S(n)$ with $\alpha \cap \beta=\emptyset$ and $\beta<\alpha$, with respect to lexicographic ordering in $S(n)$ where $\alpha=\left(i_{r}, \ldots i_{1}\right), \beta=\left(j_{s}, \ldots j_{1}\right) \in S(n)$ The pairings that we will need,

$$
\left\{F_{\alpha, \beta}: N G_{n-\sharp \alpha} \times N G_{n-\sharp \beta} \rightarrow N G_{n}:(\alpha, \beta) \in P(n), n \geq 0\right\}
$$

are given as composites by the diagram

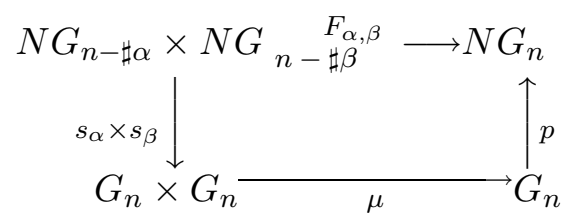

where

$s_{\alpha}=s_{i_{r}}, \ldots s_{i_{1}}: N G_{n-\sharp \alpha} \rightarrow G_{n}, \quad s_{\beta}=s_{j_{s}}, \ldots s_{j_{1}}: N G_{n-\sharp \beta} \rightarrow G_{n}$, $p: G_{n} \rightarrow N G_{n}$ is defined by composite projections $p(x)=p_{n-1} \ldots p_{0}(x)$, where

$$
p_{j}(z)=z s_{j} d_{j}(z)^{-1} \text { with } j=0,1, \ldots, n-1 \text { and }
$$

$\mu: G_{n} \times G_{n} \rightarrow G_{n}$ is given by commutator map and $\sharp \alpha$ is the number of the elements in the set of $\alpha$, similarly for $\sharp \beta$. Thus

$$
\begin{aligned}
F_{\alpha, \beta}\left(x_{\alpha}, y_{\beta}\right) & =p \mu\left(s_{\alpha} \times s_{\beta}\right)\left(x_{\alpha}, y_{\beta}\right) \\
& =p\left[s_{\alpha}\left(x_{\alpha}\right), s_{\beta}\left(x_{\beta}\right)\right] .
\end{aligned}
$$

Definition 2.2. Let $N_{n}$ or more exactly $N_{n}^{G}$ be the normal subgroup of $G_{n}$ generated by elements of the form

$$
F_{\alpha, \beta}\left(x_{\alpha}, y_{\beta}\right),
$$

where $x_{\alpha} \in N G_{n-\sharp \alpha}$ and $y_{\beta} \in N G_{n-\sharp \beta}$.

This normal subgroup $N_{n}^{G}$ depends functorially on $G$, but we will usually abbreviate $N_{n}^{G}$ to $N_{n}$, when no change of group is involved. We illustrate this normal subgroup for $n=2$ and $n=3$ to show what it looks like. 
Example 2.3. For $n=2$, assume that $\alpha=(1) \beta=(0)$ and $x, y \in N G_{1}=$ $\operatorname{ker} d_{0}$. It follows that

$$
\begin{aligned}
F_{(1)(0)}(x, y) & =p_{1} p_{0}\left(\left[s_{0} x, s_{1} y\right]\right) \\
& =p_{1}\left[s_{0} x, s_{1} y\right] \\
& =\left[s_{0} x, s_{1} y\right]\left[s_{1} y, s_{1} x\right] .
\end{aligned}
$$

is a generating element of the normal subgroup $N_{2}$.

For $n=3$, the possible pairings are the following;

$$
\begin{array}{lll}
F_{(1,0)(2)}, & F_{(2,0)(1)}, & F_{(0)(2,1)}, \\
F_{(0)(2)}, & F_{(1)(2)}, & F_{(0)(1)} .
\end{array}
$$

For all $x_{1} \in N G_{1}, y_{2} \in N G_{2}$, the corresponding generators of $N_{3}$ are:

$$
\begin{aligned}
& F_{(1,0)(2)}\left(x_{1}, y_{2}\right)=\left[s_{1} s_{0} x_{1}, s_{2} y_{2}\right]\left[s_{2} y_{2}, s_{2} s_{0} x_{1}\right] \\
& F_{(2,0)(1)}\left(x_{1}, y_{2}\right)=\left[s_{2} s_{0} x_{1}, s_{1} y_{2}\right]\left[s_{1} y_{2}, s_{2} s_{1} x_{1}\right]\left[s_{2} s_{1} x_{1}, s_{2} y_{2}\right]\left[s_{2} y_{2}, s_{2} s_{0} x_{1}\right]
\end{aligned}
$$

and for all $x_{2} \in N G_{2}, y_{1} \in N G_{1}$,

$$
F_{(0)(2,1)}\left(x_{2}, y_{1}\right)=\left[s_{0} x_{2}, s_{2} s_{1} y_{1}\right]\left[s_{2} s_{1} y_{1}, s_{1} x_{2}\right]\left[s_{2} x_{2}, s_{2} s_{1} y_{1}\right]
$$

whilst for all $x_{2}, y_{2} \in N G_{2}$,

$$
\begin{aligned}
& F_{(0)(1)}\left(x_{2}, y_{2}\right)=\left[s_{0} x_{2}, s_{1} y_{2}\right]\left[s_{1} y_{2}, s_{1} x_{2}\right]\left[s_{2} x_{2}, s_{2} y_{2}\right], \\
& F_{(0)(2)}\left(x_{2}, y_{2}\right)=\left[s_{0} x_{2}, s_{2} y_{2}\right], \\
& F_{(1)(2)}\left(x_{2}, y_{2}\right)=\left[s_{1} x_{2}, s_{2} y_{2}\right]\left[s_{2} y_{2}, s_{2} x_{2}\right] .
\end{aligned}
$$

\section{Braided Crossed Module of Groups}

In this section we will show that the descriptions of two equivalent categories: The category of braided crossed modules and the category of simplicial group with Moore complex length 2.

Crossed modules were initially defined by Whitehead in [11] as models for 2types. A crossed module $(M, P, \partial)$ is a group homomorphism $\partial: M \rightarrow P$, together with an action of $P$ on $M$ written $m^{p}$ for $p \in P$ and $m \in M$, satisfying the following conditions: for all $m, m^{\prime} \in M, p \in P$,

$$
\begin{array}{ll}
\text { CM1 }): & \partial\left(m^{p}\right)=p^{-1}(\partial m) p \\
\text { CM2) } & m^{\partial m^{\prime}}=m^{\prime-1} m m^{\prime} .
\end{array}
$$

The second condition is called Peiffer identity. 
A braided crossed modules of group(oid)s were initially defined by Brown and Gilbert in [2].

Definition 3.1. A braided crossed module of groups

$$
C_{2} \stackrel{\delta}{\rightarrow} C_{1}
$$

is a crossed module with the braiding function $\{\}:, C_{1} \times C_{1} \rightarrow C_{2}$ satisfying the following axioms:

BC1: $\left\{x, y y^{\prime}\right\}=\{x, y\}^{y^{\prime}}\left\{x, y^{\prime}\right\}$

BC2: $\left\{x x^{\prime}, y\right\}=\left\{x^{\prime}, y\right\}\{x, y\}^{x^{\prime}}$

BC3: $\delta\{x, y\}=[y, x]$

BC4: $\{x, \delta a\}=a^{-1} a^{x}$

BC5: $\{\delta b, y\}=\left(b^{-1}\right)^{y} b$

where $x, x^{\prime} y, y^{\prime} \in C_{1}$ and $a, b \in C_{2}$.

Proposition 3.2. Let $\boldsymbol{G}$ be a reduced simplicial group with Moore complex $N G$. Then the complex of groups

$$
N G_{2} / \partial_{3}\left(N G_{3} \cap D_{3}\right) \stackrel{\partial_{2}}{\longrightarrow} N G_{1}
$$

is a braided crossed module of groups. The braiding map can be defined as follows:

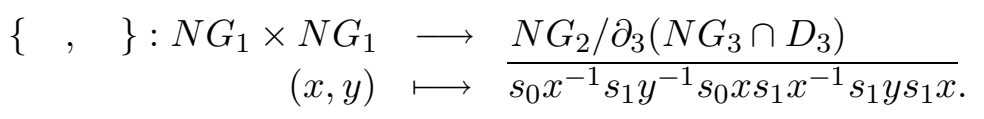

Here the right hand side denotes a coset in $N G_{2} / \partial_{3}\left(N G_{3} \cap D_{3}\right)$ represented by an element in $N G_{2}$

The two actions of $N G_{1}$ on $N G_{2} / \partial_{3}\left(N G_{3} \cap D_{3}\right)$ are given by

1. $l^{\partial_{1} m}$ corresponds to the action $s_{0}(m)^{-1} l s_{0}(m)$ and conjugation.

2. $l^{m}$ corresponds to the action $s_{1}(m)^{-1} l s_{1}(m)$.

Proof. This is a reformulation of a result of Brown and Gilbert [2]. Our aim is to show the role of the $F_{\alpha, \beta}$ in the structure. We will show that all axioms of a braided crossed module are verified. It is plainly that the morphism $\partial_{2}$ : $N G_{2} /\left(\partial_{3} N G_{3} \cap D_{3}\right) \longrightarrow N G_{1}$ is a crossed module of groups. Since every crossed module of groups is regular this construction is regular.

In the following calculations we display the elements omitting the overlines as: 
BC1: For $x_{i} \in N G_{1},(i=0,1,2$.

$$
\begin{aligned}
\left\{x_{0}, x_{1} x_{2}\right\}= & s_{0} x_{0}^{-1} s_{1} x_{2}^{-1} s_{1} x_{1}^{-1} s_{0} x_{0} s_{1} x_{0}^{-1} s_{1} x_{1} s_{1} x_{2} s_{1} x_{0} \\
= & \left(s_{0} x_{0}^{-1} s_{1} x_{2}^{-1} s_{1} x_{1}^{-1} s_{0} x_{0} s_{1} x_{0}^{-1} s_{1} x_{1}\right)\left(s_{1} x_{0} s_{0} x_{0}^{-1} s_{1} x_{2} s_{0} x_{0}\right) \\
& \left(s_{0} x_{0}^{-1} s_{1} x_{2}^{-1} s_{0} x_{0} s_{1} x_{0}^{-1}\right) s_{1} x_{2} s_{1} x_{0} \\
= & \left(s_{0} x_{0}^{-1} s_{1} x_{2}^{-1} s_{1} x_{1}^{-1} s_{0} x_{0} s_{1} x_{0}^{-1} s_{1} x_{1} s_{1} x_{0} s_{0} x_{0}^{-1} s_{1} x_{2} s_{0} x_{0}\right)\left\{x_{0}, x_{2}\right\} \\
= & s_{0} x_{0}^{-1} s_{1} x_{2}^{-1}\left(s_{0} x_{0} s_{0} x_{0}^{-1}\right)\left(s_{1} x_{1}^{-1} s_{0} x_{0} s_{1} x_{0}^{-1} s_{1} x_{1} s_{1} x_{0}\right) s_{0} x_{0}^{-1} s_{1} x_{2} s_{0} x_{0}\left\{x_{0}, x_{2}\right\} \\
= & \left(s_{0} x_{0}^{-1} s_{1} x_{2}^{-1} s_{0} x_{0}\right)\left(s_{0} x_{0}^{-1} s_{1} x_{1}^{-1} s_{0} x_{0} s_{1} x_{0}^{-1} s_{1} x_{1} s_{1} x_{0}\right) s_{0} x_{0}^{-1} s_{1} x_{2} s_{0} x_{0}\left\{x_{0}, x_{2}\right\} \\
= & s_{0} x_{0}^{-1} s_{1} x_{2}^{-1} s_{0} x_{0}\left(\left\{x_{0}, x_{1}\right\}\right) s_{0} x_{0}^{-1} s_{1} x_{2} s_{0} x_{0}\left\{x_{0}, x_{2}\right\} \\
= & \left.\left(s_{1} x_{2}^{-1}\right)^{\partial_{1} x_{0}}\left(\left\{x_{0}, x_{1}\right\}\right)\left(s_{1} x_{2}\right)^{\partial_{1} x_{0}}\right)\left(\left\{x_{0}, x_{2}\right\}\right) \\
= & \left(s_{1} x_{2}^{-1}\left\{x_{0}, x_{1}\right\} s_{1} x_{2}\right)\left\{x_{0}, x_{2}\right\} \quad \text { (since } \partial_{1}=\text { identity) } \\
= & \left\{x_{0}, x_{1}\right\}^{x_{2}}\left\{x_{0}, x_{2}\right\} . \text { (by the (2) action.) }
\end{aligned}
$$

BC2: For $y_{i} \in N G_{1},(i=0,1,2$.)

$$
\begin{aligned}
\left\{y_{0} y_{1}, y_{2}\right\}= & s_{0} y_{1}^{-1} s_{0} y_{0}^{-1} s^{1} y_{2}^{-1} s_{0} y_{0} s_{0} y_{1} s_{1} y_{1}^{-1} s_{1} y_{0}^{-1} s_{1} y_{2} s_{1} y_{0} s_{1} y_{1} \\
= & s_{0} y_{1}^{-1} s_{0} y_{0}^{-1} s^{1} y_{2}^{-1} s_{0} y_{0} s_{0} y_{1} s_{1} y_{1}^{-1}\left(s_{0} y_{0}^{-1} s_{1} y_{2} s_{0} y_{0}\right) \\
& \left(s_{0} y_{0}^{-1} s_{1} y_{2}^{-1} s_{0} y_{0}\right) s_{1} y_{0}^{-1} s_{1} y_{2} s_{1} y_{0} s_{1} y_{1} . \\
= & s_{0} y_{1}^{-1} s_{0} y_{0}^{-1} s^{1} y_{2}^{-1} s_{0} y_{0} s_{0} y_{1} s_{1} y_{1}^{-1} s_{0} y_{0}^{-1} s_{1} y_{2} s_{0} y_{0} \\
& \left(s_{0} y_{0}^{-1} s_{1} y_{2}^{-1} s_{0} y_{0} s_{1} y_{0}^{-1} s_{1} y_{2} s_{1} y_{0}\right) s_{1} y_{1} . \\
= & s_{0} y_{1}^{-1} s_{0} y_{0}^{-1} s^{1} y_{2}^{-1} s_{0} y_{0} s_{0} y_{1} s_{1} y_{1}^{-1} s_{0} y_{0}^{-1} s_{1} y_{2} s_{0} y_{0} \\
& s_{1} y_{1}\left(s_{1} y_{1}^{-1}\left\{y_{0}, y_{2}\right\} s_{1} y_{1}\right) \\
= & s_{0} y_{1}^{-1}\left(s_{1} y_{2}^{-1}\right)^{\partial_{1} y_{0}} s_{0} y_{1} s_{1} y_{1}^{-1}\left(s_{1} y_{2}\right)^{\partial_{1} y_{0}} s_{1} y_{1}\left\{y_{0}, y_{2}\right\}^{y_{1}} \text { (since the action.) } \\
= & s_{0} y_{1}^{-1} s_{1} y_{2}^{-1} s_{0} y_{1} s_{1} y_{1}^{-1} s_{1} y_{2} s_{1} y_{1}\left\{y_{0}, y_{2}\right\}^{y_{1}} \quad \text { (since } \partial_{1}=\text { identity.) } \\
= & \left\{y_{1}, y_{2}\right\}\left\{y_{0}, y_{2}\right\}^{y_{1}} .
\end{aligned}
$$

BC3:

$$
\begin{aligned}
\bar{\partial}_{2}\{x, y\} & =d_{2}\left(s_{0} x^{-1} s_{1} y^{-1} s_{0} x s_{1} x^{-1} s_{1} y s_{1} x\right) \\
& =s_{0} d_{1}\left(x^{-1}\right) y^{-1} s_{0} d_{1}(x) x^{-1} y x \\
& =\left(y^{-1}\right)^{\partial_{1} x} x^{-1} y x \quad \text { (since by action) } \\
& =y^{-1} x^{-1} y x \quad \text { (since } \partial_{1}=\text { identity) } \\
& =[y, x]
\end{aligned}
$$

\section{BC4:}

For $\alpha=(2,0), \beta=(1)$ and $x \in N G_{2}, y \in N G_{1}$ from,

$\partial_{3}\left(F_{(2,0)(1)}(y, x)\right)=\left[s_{0} y, s_{1} d_{2} x\right]\left[s_{1} d_{2} x, s_{1} y\right]\left[s_{1} y, x\right]\left[x, s_{0} y\right] \in \partial_{3}\left(N G_{3} \cap D_{3}\right)$, 


$$
\begin{aligned}
\left\{y, \bar{\partial}_{2}(x)\right\} & =\left[s_{0} y, s_{1} d_{2} x\right]\left[s_{1} d_{2} x, s_{1} y\right] \\
& \equiv\left[s_{0} y, x\right]\left[x, s_{1} y\right] \bmod \partial_{3}\left(\mathrm{NG}_{3} \cap \mathrm{D}_{3}\right) \\
& =s_{0} y^{-1} x^{-1} s_{0} y x x^{-1} s_{1} y^{-1} x s_{1} y \\
& =s_{0} y^{-1} x^{-1} s_{0} y s_{1} y^{-1} x s_{1} y \\
& \equiv s_{1} s_{0} d_{0} y^{-1} x^{-1} s_{1} s_{0} d_{0} y s_{1} y^{-1} x s_{1} y \bmod \partial_{3}\left(\mathrm{NG}_{3} \cap \mathrm{D}_{3}\right) \\
& =\left(x^{-1}\right)^{\partial_{1} y} x^{y} \\
& =x^{-1} x^{y} . \quad\left(\text { since } \partial_{1}=\text { identity }\right)
\end{aligned}
$$

BC5:

For $\alpha=(0), \beta=(2,1)$ and $x \in N G_{2}, y \in N G_{1}$ from

$$
\begin{aligned}
\partial_{3}\left(F_{(0)(2,1)}(x, y)\right)= & {\left[s_{0} d_{2} x, s_{1} y\right]\left[s_{1} y, s_{1} d_{2} x\right]\left[x, s_{1} y\right] \in \partial_{3}\left(N G_{3} \cap D_{3}\right) } \\
\left\{\bar{\partial}_{2}(x), y\right\} & =s_{0} d_{2} x^{-1} s_{1} y^{-1} s_{0} d_{2} x s_{1} d_{2} x^{-1} s_{1} y s_{1} d_{2} x \\
& =\left[s_{0} d_{2} x, s_{1} y\right]\left[s_{1} y, s_{1} d_{2} x\right] \\
& \equiv\left[s_{1} y, x\right] \bmod \partial_{3}\left(\mathrm{NG}_{3} \cap \mathrm{D}_{3}\right) \\
& =s_{1} y^{-1} x^{-1} s_{1} y x \\
& =\left(x^{-1}\right)^{y} x
\end{aligned}
$$

Therefore we show that all axioms of braided crossed module are verified.

Theorem 3.3. The category of braided crossed modules of groups is equivalent to the category of reduced simplicial groups with Moore complex of length 2.

Proof. Let $\mathbf{G}$ be a simplicial group with Moore complex of length 2. In the previous proposition, a braided crossed module

$$
N G_{2} \stackrel{\partial_{2}}{\longrightarrow} N G_{1}
$$

has already been constructed. This defines a functor from SimpGrp the category of simplicial groups to BCM the category of braided crossed modules

$$
\theta: \operatorname{SimpGrp} \rightarrow \text { BCM. }
$$

Conversely we suppose that $L \stackrel{\partial}{\longrightarrow} M$ is a braided crossed module of groups. Let $1_{M} \in M$ be identity element of the group $M$. Define $G_{0}=\left\{1_{M}\right\}$ and $G_{1}=M$. Then $\left\{G_{0}, G_{1}\right\}$ is 1-truncated simplicial group with trivial homomorphisms. 
Since $L \stackrel{\partial}{\longrightarrow} M$ is a crossed module (by using an action of $M$ on $L$ ) we can create the semidirect product $L \rtimes M$ with

$$
(l, m)\left(l^{\prime}, m^{\prime}\right)=\left(l^{m} l^{\prime}, m m^{\prime}\right) .
$$

with

$$
m^{\prime}=l^{\prime}\left\{m^{-1}, \partial\left(l^{\prime}\right)\right\} .
$$

where $\{$,$\} is braiding map and m, m^{\prime} \in M, l, l^{\prime} \in L$.

An action of $m \in M$ on $\left(l, m^{\prime}\right) \in L \rtimes M$ is given by

$$
{ }^{m}\left(l, m^{\prime}\right)=\left(l\left\{m^{-1}, \partial(l)\right\}, m m^{\prime} m^{-1}\right) .
$$

Then by using this action of $M$ on $L \rtimes M$, we can define $G_{2}=(L \rtimes M) \rtimes M$. A multiplication on $G_{2}$ is given by

$$
\left(l, m_{1}, m_{2}\right) \cdot\left(l^{\prime}, m_{1}^{\prime}, m_{2}^{\prime}\right)=\left(l l^{\prime}\left\{m_{2}^{-1} m_{1}^{-1}, \partial\left(l^{\prime}\right)\right\}, m_{1} m_{2} m_{1}^{\prime} m_{2}^{-1}, m_{2} m_{2}^{\prime}\right)
$$

We will show that $G_{2}$ is a group. It is clear that $\left(1_{L}, 1_{M}, 1_{M}\right)$ is identity element of $G_{2}$, because

$$
\begin{aligned}
\left(l, m_{1}, m_{2}\right) \cdot\left(1_{L}, 1_{M}, 1_{M}\right) & =\left(l 1_{M}\left\{m_{2}^{-1} m_{1}^{-1}, \partial\left(1_{L}\right)\right\}, m_{1} m_{2} 1_{M} m_{2}^{-1}, m_{2} 1_{M}\right) \\
& =\left(l\left\{m_{2}^{-1} m_{1}^{-1}, 1_{M}\right\}, m_{1}, m_{2}\right) \\
& =\left(l 1_{L}, m_{1}, m_{2}\right) \\
& =\left(l, m_{1}, m_{2}\right) .
\end{aligned}
$$

The invers element of $\left(l, m_{1}, m_{2}\right) \in G_{2}$ is given by

$$
\left(l, m_{1}, m_{2}\right)^{-1}=\left(l^{-1}\left\{m_{1} m_{2}, \partial\left(l^{-1}\right)\right\}, m_{2}^{-1} m_{1}^{-1} m_{2}, m_{2}^{-1}\right)
$$

Indeed,

$$
\begin{aligned}
\left(l, m_{1}, m_{2}\right) .\left(l, m_{1}, m_{2}\right)^{-1}= & \left(l, m_{1}, m_{2}\right)\left(l^{-1}\left\{m_{1} m_{2}, \partial\left(l^{-1}\right)\right\}, m_{2}^{-1} m_{1}^{-1} m_{2}, m_{2}^{-1}\right) \\
= & \left(l l^{-1}\left\{m_{1} m_{2}, \partial\left(l^{-1}\right)\right\}\left\{m_{2}^{-1} m_{1}^{-1}, \partial\left(l^{-1}\left\{m_{1} m_{2}, \partial\left(l^{-1}\right)\right\}\right)\right\},\right. \\
& \left.m_{1} m_{2} m_{2}^{-1} m_{1}^{-1} m_{2} m_{2}^{-1}, m_{2} m_{2}^{-1}\right) \\
= & \left(l\left(^{m_{2}^{-1} m_{1}^{-1}} l^{-1}\right)\left({ }^{m_{1} m_{2}} l\right)\left({ }^{m_{1} m_{2}}\left({ }^{m_{2}^{-1} m_{1}^{-1}} l^{-1}\right)\right), 1_{M}, 1_{M}\right) \\
= & \left(l l^{-1}, 1_{M}, 1_{M}\right) \\
= & \left(1_{L}, 1_{M}, 1_{M}\right) .
\end{aligned}
$$

It is clear that the multiplication on $G_{2}$ is associative. Indeed, for

$$
\begin{aligned}
& x_{0}=\left(l, m_{1}, m_{2}\right) \\
& x_{1}=\left(l^{\prime}, m_{1}^{\prime}, m_{2}^{\prime}\right) \\
& x_{2}=\left(l^{\prime \prime}, m_{1}^{\prime \prime}, m_{2}^{\prime \prime}\right),
\end{aligned}
$$




$$
\begin{aligned}
x_{0}\left(x_{1} x_{2}\right) & =\left(l, m_{1}, m_{2}\right) \cdot\left[\left(l^{\prime}, m_{1}^{\prime}, m_{2}^{\prime}\right) \cdot\left(l^{\prime \prime}, m_{1}^{\prime \prime}, m_{2}^{\prime \prime}\right)\right] \\
& =\left(l, m_{1}, m_{2}\right) \cdot\left(l^{\prime}\left(m_{1}^{\prime} m_{2}^{\prime} l^{\prime \prime}\right), m_{1}^{\prime} m_{2}^{\prime} m_{1}^{\prime \prime}\left(m_{2}^{\prime}\right)^{-1}, m_{2}^{\prime} m_{2}^{\prime \prime}\right) \\
& =\left(l\left(^{m_{1} m_{2}}\left(l^{\prime m_{1}^{\prime} m_{2}^{\prime}} l^{\prime \prime}\right)\right), m_{1} m_{2}\left(m_{1}^{\prime} m_{2}^{\prime} m_{1}^{\prime \prime}\left(m_{2}^{\prime}\right)^{-1}\right) m_{2}^{-1}, m_{2} m_{2}^{\prime} m_{2}^{\prime \prime}\right) \\
& =\left(l\left(^{m_{1} m_{2}} l^{\prime}\right)\left({ }^{m_{1} m_{2} m_{1}^{\prime} m_{2}^{\prime}} l^{\prime \prime}\right), m_{1} m_{2} m_{1}^{\prime} m_{2}^{\prime} m_{1}^{\prime \prime}\left(m_{2}^{\prime}\right)^{-1} m_{2}^{-1}, m_{2} m_{2}^{\prime} m_{2}^{\prime \prime}\right)
\end{aligned}
$$

and

$$
\begin{aligned}
\left(x_{0} x_{1}\right) x_{2}= & {\left[\left(l, m_{1}, m_{2}\right) \cdot\left(l^{\prime}, m_{1}^{\prime}, m_{2}^{\prime}\right)\right]\left(l^{\prime \prime}, m_{1}^{\prime \prime}, m_{2}^{\prime \prime}\right) } \\
= & {\left[\left(l\left(^{m_{1} m_{2}} l^{\prime}\right), m_{1} m_{2} m_{1}^{\prime} m_{2}^{-1}, m_{2} m_{2}^{\prime}\right)\right] \cdot\left(l^{\prime \prime}, m_{1}^{\prime \prime}, m_{2}^{\prime \prime}\right) } \\
= & {\left[\left(l\left({ }^{m_{1} m_{2}} l^{\prime}\right)\left(m_{1} m_{2} m_{1}^{\prime} m_{2}^{-1} m_{2} m_{2} l^{\prime \prime}\right), m_{1} m_{2} m_{1}^{\prime} m_{2}^{-1} m_{2} m_{2}^{\prime} m_{1}^{\prime \prime}\right.\right.} \\
& \left.\left.\left(m_{2} m_{2}^{\prime}\right)^{-1}, m_{2} m_{2}^{\prime} m_{2}^{\prime \prime}\right)\right] \\
= & \left(l\left({ }^{m_{1} m_{2}} l^{\prime}\right)\left(m_{1} m_{2} m_{1}^{\prime} m_{2}^{\prime} l^{\prime \prime}\right), m_{1} m_{2} m_{1}^{\prime} m_{2}^{\prime} m_{1}^{\prime \prime}\left(m_{2}^{\prime}\right)^{-1} m_{2}^{-1}, m_{2} m_{2}^{\prime} m_{2}^{\prime \prime}\right) .
\end{aligned}
$$

Therefore we have

$$
\left(x_{0} x_{1}\right) x_{2}=x_{0}\left(x_{1} x_{2}\right) .
$$

We have homomorphisms

$$
\begin{aligned}
d_{0}^{2}\left(l, m_{1}, m_{2}\right) & =m_{1} \\
d_{1}^{2}\left(l, m_{1}, m_{2}\right) & =m_{1} m_{2} \\
d_{2}^{2}\left(l, m_{1}, m_{2}\right) & =m_{2} \\
s_{0}^{1}\left(m_{1}\right) & =\left(1_{L}, m_{1}, 1_{M}\right) \\
s_{1}^{1}\left(m_{1}\right) & =\left(1_{L}, 1_{M}, m_{1}\right)
\end{aligned}
$$

These maps satisfy the simplicial identities.

Thus we have a 2-truncated simplicial group

$$
\left\{G_{0}, G_{1}, G_{2}\right\} \text {. }
$$

There is a $\operatorname{cosk}_{2}$ functor from the category of 2- truncated simplicial groups to that of simplicial groups.

We have that $\partial_{3}\left(N G_{3} \cap D_{3}\right)$ is the product $\left[\operatorname{ker} d_{2}, \operatorname{ker} d_{0} \cap \operatorname{ker} d_{1}\right]$, $\left[\operatorname{ker} d_{1}, \operatorname{ker} d_{0}\right.$ $\left.\cap \operatorname{ker} d_{2}\right],\left[\operatorname{ker} d_{1} \cap \operatorname{ker} d_{2}, \operatorname{ker} d_{0}\right],\left[\operatorname{ker} d_{0} \cap \operatorname{ker} d_{2}, \operatorname{ker} d_{0} \cap \operatorname{ker} d_{1}\right],\left[\operatorname{ker} d_{0} \cap \operatorname{ker} d_{1}, \operatorname{ker} d_{1} \cap\right.$ $\left.\operatorname{ker} d_{2}\right]$ and

$\left[\operatorname{ker} d_{0} \cap \operatorname{ker} d_{1}, \operatorname{ker} d_{1} \cap \operatorname{ker} d_{2}\right]$. Now we show that $\partial_{3}\left(N G_{3}\right)=$ identity. For this we use the functions $F_{\alpha, \beta}$. 
Step 1 : For $\alpha=(1,0), \beta=(2)$ and $x=m \in N G_{1}, y \in \operatorname{ker} d_{0} \cap \operatorname{ker} d_{1}$, where $y=\left(l, 1_{M}, 1_{M}\right)$. Then

$$
\begin{aligned}
\partial_{3}\left(F_{(1,0)(2)}(x, y)\right) & =\left[s_{1} s_{0} d_{1} m,\left(l, 1_{M}, 1_{M}\right)\right]\left[\left(l, 1_{M}, 1_{M}\right), s_{0} m\right] \\
& =\left(l, 1_{M}, 1_{M}\right)\left(l, m, 1_{M}\right)\left(l^{-1}, 1_{M}, 1_{M}\right)\left(l, m^{-1}, 1_{M}\right) \\
& =\left(l, m, 1_{M}\right)\left(l^{-1}, m^{-1}, 1_{M}\right) \\
& =\left(l^{m} l^{-1}, 1_{M}, 1_{M}\right) \in\left[\operatorname{ker} d_{2}, \operatorname{ker} d_{0} \cap \operatorname{ker} d_{1}\right] .
\end{aligned}
$$

Step 2 : For $\alpha=(2,0), \beta=(1)$ and $x=m \in N G_{1}, y \in \operatorname{ker} d_{0} \cap \operatorname{ker} d_{1}$, where $y=\left(l, 1_{M}, 1_{M}\right)$. Then

$$
\begin{aligned}
\partial_{3}\left(F_{(2,0)(1)}(x, y)\right)= & {\left[s_{0} m, s_{1} d_{2}\left(l, 1_{M}, 1_{M}\right)\right]\left[s_{1} d_{2}\left(l, 1_{M}, 1_{M}\right), s_{1} m\right] } \\
& {\left[s_{1} m,\left(l, 1_{M}, 1_{M}\right)\right]\left[\left(l, 1_{M}, 1_{M}\right), s_{0} m\right] } \\
= & \left({ }^{m} l, 1_{M}, m\right)\left(1_{L}, m, m^{-1}\right)\left(l^{-1}, m^{-1}, 1_{M}\right) \\
= & \left({ }^{m} l, 1_{M}, m\right)\left(l^{-1}, 1_{M}, m^{-1}\right) \\
= & \left(1_{L}, 1_{M}, 1_{M}\right) \in\left[\operatorname{ker} d_{1}, \operatorname{ker} d_{0} \cap \operatorname{ker} d_{2}\right] .
\end{aligned}
$$

Step 3 : For $\alpha=(2,1), \beta=(0)$ and $x=m \in N G_{1}, y=\left(l, 1_{M}, 1_{M}\right) \in N G_{2}$. Then

$$
\begin{aligned}
\partial_{3}\left(F_{(2,1)(0)}(x, y)\right) & =\left[s_{1} m, s_{0} d_{2}\left(l, 1_{M}, 1_{M}\right)\right]\left[s_{1} d_{2}\left(l, 1_{M}, 1_{M}\right), s_{1} m\right]\left[s_{1} m,\left(l, 1_{M}, 1_{M}\right)\right] \\
& =\left(1_{L}, 1_{M}, m\right)\left(l, 1_{M}, 1_{M}\right)\left(1_{L}, 1_{M}, m^{-1}\right)\left(l^{-1}, 1_{M}, 1_{M}\right) \\
& =\left({ }^{m} l, 1_{M}, m\right)\left({ }^{-1} l^{-1}, 1_{M}, m^{-1}\right) \\
& =\left({ }^{m} l l^{-1}, 1_{M}, 1_{M}\right) \in\left[\operatorname{ker} d_{1} \cap \operatorname{ker} d_{2}, \operatorname{ker} d_{0}\right]
\end{aligned}
$$

Step 4 : For $\alpha=(2), \beta=(1)$,let $x=\left(l^{\prime}, 1_{M}, 1_{M}\right), y=\left(l, 1_{M}, 1_{M}\right)$ be any elements of $N G_{2}$. Then

$$
\begin{aligned}
\partial_{3}\left(F_{(2)(1)}(x, y)\right) & =\left[x, s_{1} d_{2} y\right][y, x] \\
& =\left(l, 1_{M}, 1_{M}\right)\left(l^{\prime}, 1_{M}, 1_{M}\right)\left(l^{-1}, 1_{M}, 1_{M}\right)\left(l^{\prime-1}, 1_{M}, 1_{M}\right) \\
& =\left(l l^{\prime} l^{-1} l^{\prime-1}, 1_{M}, 1_{M}\right) \in\left[\operatorname{ker} d_{0} \cap \operatorname{ker} d_{2}, \operatorname{ker} d_{0} \cap \operatorname{ker} d_{1}\right] .
\end{aligned}
$$

Step 5 : For $\alpha=(2), \beta=(0)$,let $x=\left(l^{\prime}, 1_{M}, 1_{M}\right), y=\left(l, 1_{M}, 1_{M}\right)$ be any elements of $N G_{2}$. Then

$$
\begin{aligned}
\partial_{3}\left(F_{(2)(0)}(x, y)\right) & =\left[x, s_{0} d_{2} y\right] \\
& =\left(l^{\prime}, 1_{M}, 1_{M}\right)\left(l^{\prime-1}, 1_{M}, 1_{M}\right) \\
& =\left(1_{L}, 1_{M}, 1_{M}\right) \in\left[\operatorname{ker} d_{0} \cap \operatorname{ker} d_{1}, \operatorname{ker} d_{1} \cap \operatorname{ker} d_{2}\right] .
\end{aligned}
$$


Step 6: For $\alpha=(1), \beta=(0)$,let $x=\left(l^{\prime}, 1_{M}, 1_{M}\right), y=\left(l, 1_{M}, 1_{M}\right)$ be any elements of $N G_{2}$. Then

$$
\begin{aligned}
\partial_{3}\left(F_{(1)(0)}(x, y)\right) & =\left[s_{1} d_{2} x, s_{0} d_{2} y\right]\left[s_{1} d_{2} y, s_{1} d_{2} x\right][x, y] \\
& =\left(l^{\prime}, 1_{M}, 1_{M}\right)\left(l, 1_{M}, 1_{M}\right)\left(l^{\prime-1}, 1_{M}, 1_{M}\right)\left(l^{-1}, 1_{M}, 1_{M}\right) \\
& =\left(l^{\prime} l l^{\prime-1} l^{-1}, 1_{M}, 1_{M}\right) \in\left[\operatorname{ker} d_{0} \cap \operatorname{ker} d_{1}, \operatorname{ker} d_{1} \cap \operatorname{ker} d_{2}\right] .
\end{aligned}
$$

Since

$$
\begin{aligned}
\partial_{3}\left(N G_{3}\right)= & {\left[\operatorname{ker} d_{2}, \operatorname{ker} d_{0} \cap \operatorname{ker} d_{1}\right]\left[\operatorname{ker} d_{1}, \operatorname{ker} d_{0} \cap \operatorname{ker} d_{2}\right] } \\
& {\left[\operatorname{ker} d_{1} \cap \operatorname{ker} d_{2}, \operatorname{ker} d_{0}\right]\left[\operatorname{ker} d_{0} \cap \operatorname{ker} d_{2}, \operatorname{ker} d_{0} \cap \operatorname{ker} d_{1}\right] } \\
& {\left[\operatorname{ker} d_{0} \cap \operatorname{ker} d_{2}, \operatorname{ker} d_{1} \cap \operatorname{ker} d_{2}\right]\left[\operatorname{ker} d_{0} \cap \operatorname{ker} d_{1}, \operatorname{ker} d_{1} \cap \operatorname{ker} d_{2}\right] . }
\end{aligned}
$$

Therefore for $\tau \in \partial_{3}\left(N G_{3}\right)$,

$$
\begin{aligned}
\tau & =\left(l^{m} l^{-1}, 1_{M}, 1_{M}\right)\left(1_{L}, 1_{M}, 1_{M}\right)\left({ }^{m} l l^{-1}, 1_{M}, 1_{M}\right) \\
& =\left(l l^{\prime} l^{-1} l^{-1}, 1_{M}, 1_{M}\right)\left(1_{L}, 1_{M}, 1_{M}\right)\left(l^{\prime} l l^{-1} l^{-1}, 1_{M}, 1_{M}\right) \\
& =\left(l^{m}\left(l^{-1}\right)^{m} l l^{-1}, 1_{M}, 1_{M}\right)\left(l l^{\prime} l^{-1} l^{\prime-1} l^{\prime} l l^{\prime-1} l^{-1}, 1_{M}, 1_{M}\right) \\
& =\left(1_{L}, 1_{M}, 1_{M}\right) .
\end{aligned}
$$

\section{REFERENCES}

1. Z. Arvasi and T. Porter, Freenes Conditions for 2-Crossed Modules of Commutative Algebras, Theory and Applications of Categories.

2. R. Brown and N. D. Gilbert, Algebraic Models of 3-Types and Automorphism Structures for Crossed Modules Proc. London Math. Soc. (3) 59 (1989), 51-73.

3. P. Carrasco, Complejos Hipercruzados Cohomologia y Extensiones, Ph.D. Thesis, Universidad de Granada, (1987).

4. P. Carrasco and A. M. Cegarra, Group-theoretic algebraic models for homotopy types, Journal Pure Appl. Algebra, 75 (1991), 195-235.

5. D. Conduché, Modules croisés géneralisés de longueur 2, Journal Pure Appl. Algebra, 34 (1984), 155-178.

6. J. Duskin, Simplicials Methods and the Interpretation of Triple cohomology, Memoirs A.M.S. Vol. 3, 163 (1975).

7. A. Mutlu and T. Porter, Freenes Conditions for 2-Crossed Modules and Complexes, Theory and Applications of Categories, Vol. 4, 8 (1998), 174-194. 
8. A. Joyal and R. Street, Braided Monodial Categories, Macquarie Mathematics Report 860081 Macquarie University, 1986.

9. A. Mutlu and T. Porter, Application of Peiffer Pairings in the Moore Complex of Simplicial Group, Theory and Applications of Categories, Vol. 4, 8 (1998), 174194.

10. J. H. C. Whitehead, Combinatorial Homotopy I and II, Bull. Amer. Math. Soc. 55 (1949), 231-245 and 453-496.

Z. Arvasi, M. Koçak and E. Ulualan Osmangazi University,

Department of Mathematics,

26480, Eskişehir,

Turkey 